\title{
Signal and Image Reconstruction in Undergraduate Education: Tools for Assessing and Comparing a Set of Algorithms
}

\author{
MANUEL J. C. S. REIS, ${ }^{1}$ PAULO J. S. G. FERREIRA ${ }^{2}$ \\ ${ }^{1}$ Departamento de Engenharias/CETAV, Universidade de Trás-os-Montes e Alto Douro, 5000-911 Vila Real, Portugal \\ ${ }^{2}$ Departamento Electrónica e Telecomunicações/IEETA, Universidade de Aveiro, 3810-193 Aveiro, Portugal
}

Received 26 September 2003; accepted 30 May 2004

\begin{abstract}
This paper discusses signal and image reconstruction in connection with undergraduate electrical engineering education. It briefly reviews some of the basic signal and image reconstruction techniques, and presents two Java applets that have been found useful in the context. The applets have easy-to-use, friendly interfaces, and can be used as tools to teach reconstruction techniques or as a laboratory to study the applicability of the methods to real world signals and images. The students can do simulations with their own data (signals or images) on any computer platform. @ 2004 Wiley Periodicals, Inc. Comput Appl Eng Educ 12: 242-248, 2004; Published online in Wiley InterScience (www.interscience.wiley.com); DOI 10.1002/cae.20021
\end{abstract}

Keywords: signal reconstruction; interpolation; java applets

\section{INTRODUCTION}

\section{Motivation and Background}

Signal and image reconstruction are among the problems often faced by anyone working in the broad

Correspondence to M. J. C. S. Reis (mcabral@utad.pt). (C) 2004 Wiley Periodicals Inc. field of multidimensional signal processing. A large fraction of the signal processing literature is devoted to problems which fall in the scope of multidimensional signal reconstruction, and which includes sampling theory, interpolation, extrapolation, signal and image conditioning, interactive image repair, deconvolution and other inverse problems, reconstruction in tomography, filter design, and much more. 
For this reason, an introductory course focusing on signal reconstruction would be an welcome addition to the background of Electrical Engineering students, specially to those interested in telecommunications, information theory, or signal/image processing in general. In this paper, we report on our experience in teaching such courses at the Electronics and Telecommunications Department of the University of Aveiro and at the University of Trás-os-Montes e Alto Douro.

Solving problems while experimenting and comparing the available algorithms is of fundamental importance in the learning process. In this paper, we present two applets intended as tools to help the students do that. We felt a need for tools that could be easily used outside the classroom, easily updated and/or maintained, and were platform independent. The applets allow the student to try several algorithms on their own data, and then compare the results in an easy-to-use environment that is similar to a laboratory.

We believe that a small introduction to signal reconstruction will help to clarify the ideas that lead to the development of the applets. The next sections are dedicated to this.

\section{Sampling and Finite-Dimensional Problems}

One of the most well-known signal reconstruction problems is the one solved by the classical sampling theorem (see Ref. 1 for some recent research and a historical overview). Clearly, the classical sampling theorem can be interpreted in the context of signal reconstruction. It expresses the possibility of interpolating the band-limited signal $f(t)$ from its samples $f(k T), k \in \mathbb{Z}$, theoretically without error. A signal $f(t)$ is band-limited to $[-\sigma, \sigma]$, if it can be written as

$$
f(t)=\frac{1}{\sqrt{2 \pi}} \int_{-\sigma}^{\sigma} \hat{f}(w) e^{j w t} \mathrm{~d} w
$$

where $\hat{f}$ denotes the Fourier transform of $f$,

$$
\hat{f}(w)=\frac{1}{\sqrt{2 \pi}} \int_{-\infty}^{\infty} f(t) e^{-j w t} \mathrm{~d} t .
$$

For $W \geq(\sigma / \pi)$, the signal can be recovered from its samples $f(k / W), k \in \mathbb{Z}$, as follows:

$$
f(t)=\sum_{k=-\infty}^{+\infty} f\left(\frac{k}{W}\right) \operatorname{sinc}(W t-k), \quad t \in \mathbb{R},
$$

where $\operatorname{sinc}(t)=\sin (\pi t) / \pi t, t \neq 0$ and $\operatorname{sinc}(0)=1$.

If the sampling rate is higher than the minimum rate (known as the Nyquist's rate), we say that we are oversampling the signal. In practical situations this is often the case. In fact, the reconstruction of signals sampled at the minimum rate (critically sampled), although theoretically possible, requires impractical reconstruction procedures.

A side effect of the oversampling is the possibility of recovering some of the samples from the remaining ones $[3,14]$. The oversampled sampling representation is

$$
f(t)=r \sum_{k=-\infty}^{+\infty} f\left(\frac{k}{W}\right) \operatorname{sinc}[r(W t-k)],
$$

where $r=\sigma /(W \pi), 0<r<1$, is known as the oversampling parameter. It leads [3] to a set of linear equations for a set of unknown samples. This means that, in the oversampled case, the loss of a (finite) set of samples is no obstacle to the total recovery of the signal.

In practical situations, the need to know all the samples $f(k / W)$ for $k \in \mathbb{Z}$ raises problems. Computer simulations require finitely many data, and a way around this difficulty is to consider $n$-periodic signals. These signals are defined by a finite number of samples (exactly $n$ samples), the remaining samples being obtained by periodic repetition of the first $n$.

In the finite-dimensional setting the natural harmonic analysis tool is the discrete Fourier transform (DFT). The algorithms discussed below are based on it. They may be further grouped in two classes: $n$-dimensional algorithms (or maximum dimension algorithms), where $n$ is the number of samples of the signals, and $p$-dimensional algorithms (or minimum dimension algorithms), where $p$ denotes the number of unknown samples (in the time or frequency domains). Methods such as the Papoulis-Gerchberg iteration [11,15], and the method of alternating projections [20] lead to algorithms belonging to the first class. The formulations proposed in References 5,10 and 12,19, belong to the second class. They lead to sets of linear equations that directly determine the missing time-domain samples, or the DFT coefficients of the signal.

\section{Papoulis-Gerchberg and Alternating Projections Algorithms}

The Papoulis-Gerchberg algorithm was independently proposed in References 15 and 11 to solve the band-limited continuous-time, finite-energy extrapolation problem. The finite-dimensional version was studied in References 13 and 4. This algorithm can be seen as a particular case of the method of alternating projections [20]. For a detailed convergence analysis that includes the effect of the distribution of the missing samples see Reference 4. 
Regardless of the number of missing samples, each iteration of the algorithm acts on vectors of dimension $n$, where $n$ is the total number of samples of the signal. If the signals are regarded as $n$-periodic, $n$ is the number of samples in one signal period.

The fact that the vectors involved in the reconstruction process are $n$-dimensional seems wasteful, from the viewpoint of memory and computational needs, particularly when the number of missing samples is much smaller than $n$. This remains true even when simple convergence acceleration techniques are used (such as relaxation).

\section{Minimum Dimension Algorithms}

The methods of minimum dimension $[5,10]$ manipulate vectors with dimension determined by the number of unknown samples. They come in two flavors: the time-domain methods lead directly to equations for the unknown samples, whereas the frequency-domain methods lead to equations for the unknown DFT samples. Note that by hypothesis, the signals are band-limited, meaning that a subset of the DFT samples is known to be zero. The relation between this way of looking at the problem and the methods discussed in References 12,19 (which are in fact of minimum-dimension in the frequency domain) was discussed in Reference 7.

The size and structure of the time-domain equations is different from the size and structure of the frequency-domain equations. They can be solved noniteratively, using any standard method, or iteratively, using stationary methods such as SOR, or semiiterative methods such as conjugate gradients. This leads to a variety of distinct possibilities for the solution of the decoding problem.

In general, iterative methods have one advantage: any a priori knowledge concerning the solution can readily be incorporated in the algorithm. Since an approximation to the solution is available at each iteration, it is a very simple matter to incorporate known and possibly nonlinear constraints in the algorithm. If the constraint operator is $T$, and $f^{(i)}$ is the approximation at the end of iteration $i$, then $f^{(i)}$ should simply be replaced with $T f^{(i)}$.

This usually speeds up the convergence and leads to better reconstructions. When the constraints define convex sets, the result is a POCS-like iteration. The analytical tools include convex and alternating projections theory, and fixed point theorems.

Incorporating a priori knowledge in a noniterative algorithm is in general much more difficult, if not impossible. However, the noniterative methods may work faster. The situation is far from simple, however, and one word of caution is necessary. A careful study of the decoding algorithms shows that there is no "best algorithm" overall [8]. The fastest algorithm is a function of the problem. It depends on the value of $n$ (number of samples), on the dimension of the code subspace $2 M+1$ (number of non-null harmonics in the DFT), and also on the number and distribution of the missing samples. The break-even point that determines when one of the algorithms starts being preferable to others may also depend on the architecture of the computer (cache size, for example, is an important factor).

It might make more sense to discuss time-domain versus frequency-domain formulations than iterative versus noniterative solutions. In fact, both formulations lead to equations that can be solved iteratively or noniteratively.

Generally speaking, the methods (iterative or noniterative) that spring from the time-domain formulation tend to be better when the number of missing samples is relatively small and the oversampling is moderate. On the other hand, iterative or noniterative solutions obtained from the frequency-domain formulation may be preferable when the number of missing samples is relatively large, and the degree of oversampling higher.

The error pattern is also a crucial factor and should be considered. The frequency-domain equations are always Toeplitz and require complex arithmetic. The time-domain equations are Toeplitz only if the pattern of missing samples is regular or contiguous, and require only real arithmetic.

Weighting all these factors is not easy, but is crucial to obtain effective numerical algorithms for the solution of a specific problem or a class of problems. For specific results concerning the performance and choice of the reconstruction algorithm see Reference [8].

\section{SOLVING THE RECONSTRUCTION PROBLEM}

The minimum-dimension equations in time $[3,6]$ are of the form

$$
\mathbf{u}=\mathbf{S u}+\mathbf{h},
$$

where the set $\mathcal{U}=\left\{i_{0}, i_{1}, \ldots, i_{p-1}\right\}$ contains the indices of the unknown samples. The matrix $\mathbf{S}$ and the vector $\mathbf{h}$ are known. In fact,

$$
h_{j}=\sum_{k \notin \mathcal{U}} f(k T) K^{r}\left(i_{j}-k\right), \quad(0 \leq j<p),
$$


and

$$
S_{j k}=K^{r}\left(i_{j}-i_{k}\right), \quad(0 \leq j, k<p),
$$

where $K^{r}(x)=r \operatorname{sinc}(r x)$, i.e., the usual interpolation kernel.

It is also possible to replace these kernels by $K^{r}=r \varphi_{i}(r x), i=1,2,6,7$, (the interpolation kernels proposed by Butzer et al. in Reference 2), where

$$
\begin{aligned}
\varphi_{1}(t)= & M_{2}(t) \\
\varphi_{2}(t)= & 4 M_{3}(t)-3 M_{4}(t) \\
\varphi_{6}(t)= & M_{4}(t)+\frac{1}{3} M_{2}(t)-\frac{1}{6}\left\{M_{2}(t+1)+M_{2}(t-1)\right\} \\
\varphi_{7}(t)= & 4 M_{4}(t)+\frac{1}{2}\left\{M_{4}(t+1)+M_{4}(t-1)\right\} \\
& -2\left\{M_{5}\left(t+\frac{1}{2}\right)+M_{5}\left(t-\frac{1}{2}\right)\right\} .
\end{aligned}
$$

A study of these functions as interpolation kernels can be found in Reference 17, where it is shown its superior behaviour compared to the traditional sinc, when $r \approx 1$.

In the two-dimensional case, these kernels may be used in the following way:

$$
\begin{aligned}
h_{i j}= & \sum_{k \notin \mathcal{U}} \sum_{l \notin \mathcal{U}} f_{k l} K_{R}^{r_{R}}\left(\rho_{i}-\rho_{k}\right) K_{C}^{r_{C}}\left(\lambda_{j}-\lambda_{l}\right), \\
& (0 \leq i, j<p),
\end{aligned}
$$

and

$$
S_{i j}=K_{R}^{r_{R}}\left(\rho_{i}-\rho_{j}\right) K_{C}^{r_{C}}\left(\lambda_{i}-\lambda_{j}\right), \quad(0 \leq i, j<p),
$$

with

$$
\begin{aligned}
& K_{R}^{r_{R}}=\left\{\begin{array}{l}
r_{R} \operatorname{sinc}\left(r_{R} x\right), \text { or } \\
r_{R} \varphi\left(r_{R} x\right)
\end{array},\right. \\
& K_{C}^{r_{C}}=\left\{\begin{array}{l}
r_{C} \operatorname{sinc}\left(r_{C} x\right), \text { or } \\
r_{C} \varphi\left(r_{C} x\right)
\end{array},\right.
\end{aligned}
$$

and

$$
\mathcal{U}=\left\{\left(\rho_{0}, \lambda_{0}\right),\left(\rho_{1}, \lambda_{1}\right), \ldots,\left(\rho_{p-1}, \lambda_{p-1}\right)\right\} .
$$

Equation 2 suggests the simple iteration

$$
\mathbf{u}^{(i+1)}=\mathbf{S} \mathbf{u}^{(i)}+\mathbf{h},
$$

but a number of other iteration techniques can be used. The equation can also be solved noniteratively,

$$
\mathbf{u}=(\mathbf{I}-\mathbf{S})^{-1} \mathbf{h},
$$

and it is known that if there are enough data (that is, if the number of known samples exceeds the dimension of the subspace of band-limited signals) then the required inverse exists and the iterative method will converge.

The method of conjugate gradients is among the most efficient method for solving these problems. Among the noniterative methods, the Cholevsky factorization often leads to good execution times, and the singular value decomposition (SVD) to increased precision $[16,18]$. Definite answers depend on the details of the problem [9], as discussed before.

All of the assertions and methods mentioned can be verified with the help of the tools discussed in the next section.

\section{Filling the Linear Equations System}

In practice, $\mathbf{h}$ is not found using Equation 3. Instead, a procedure based on the FFT is used. First, the unknown samples in the observed signal (clone in the listing 1) are replaced with zeros. The signal is then low-pass-filtered to the same bandwidth of the original signal. The values left at the unknown locations are the values of $\mathbf{h}$.

To fill the matrix $\mathbf{S}$ we use two auxiliary variables in order to clarify (dummy) and speed up (lbn) the computations.

The Java code fragment in listing 1 serves this end. This helps the students to better understand the problem in hands. Note that the code is not purely object-oriented, but we think that it is easier to understand as it stands.

\section{THE APPLETS}

The two Java applets are available at http://www.utad.pt/ mcabral.

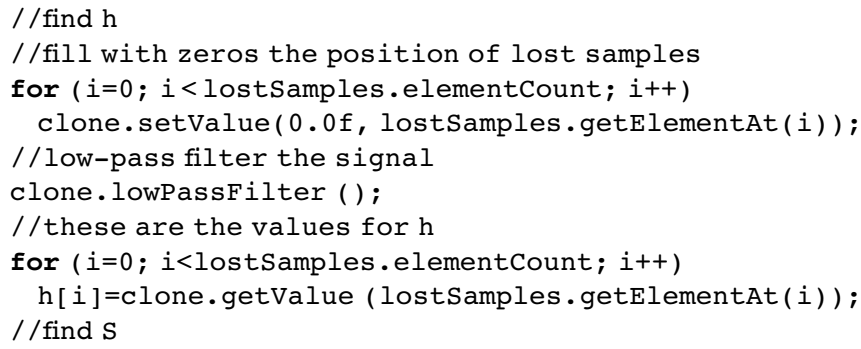




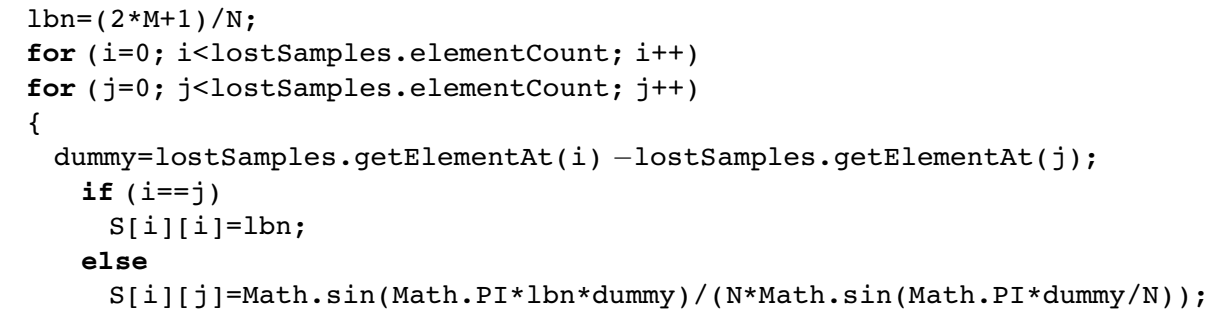

Listing 1 Java code sample to find $\mathbf{h}$ and $\mathbf{S}$.

One is dedicated to image reconstruction problems, and the other is for one-dimensional signal reconstruction. They implement the previously discussed methods, and are intended to be used as signal and image reconstruction teaching tools. They can be used inside or outside the classroom, as an experimental laboratory, or as a means to test the methods with concrete reconstruction problems.

The object-oriented philosophy leads to code that is easier to maintain, and easier to extend. This is an important point since it reduces the effort necessary to include new algorithms. The following sections describe the applets in more detail.

\section{Signal Reconstruction Applet}

The applet has a default signal set that can be used by the student. However, the student may also export its own signals simply by filling an appropriate form. The signal format must be AU or WAVE. These are two of the most commonly used encoding formats (a great number of conversion tools between different encoding formats are available as freeware or shareware on the internet). In addition to this, it is also possible to select the signal segment that will be used during the simulations, as well as the oversampling parameter $r$ (by specifying the number of non-null harmonics of the DFT of the signal).

A variety of methods for the solution of the linear equations can be tried, including iterative and noniterative methods. The iterative methods stop when a certain number of iterations has been completed, or when $\left\|\mathbf{u}_{i}-\mathbf{u}_{i-1}\right\|$ falls below a certain threshold. The student may experiment with these parameters, and may also set the value of a relaxation constant, used by some of the methods.

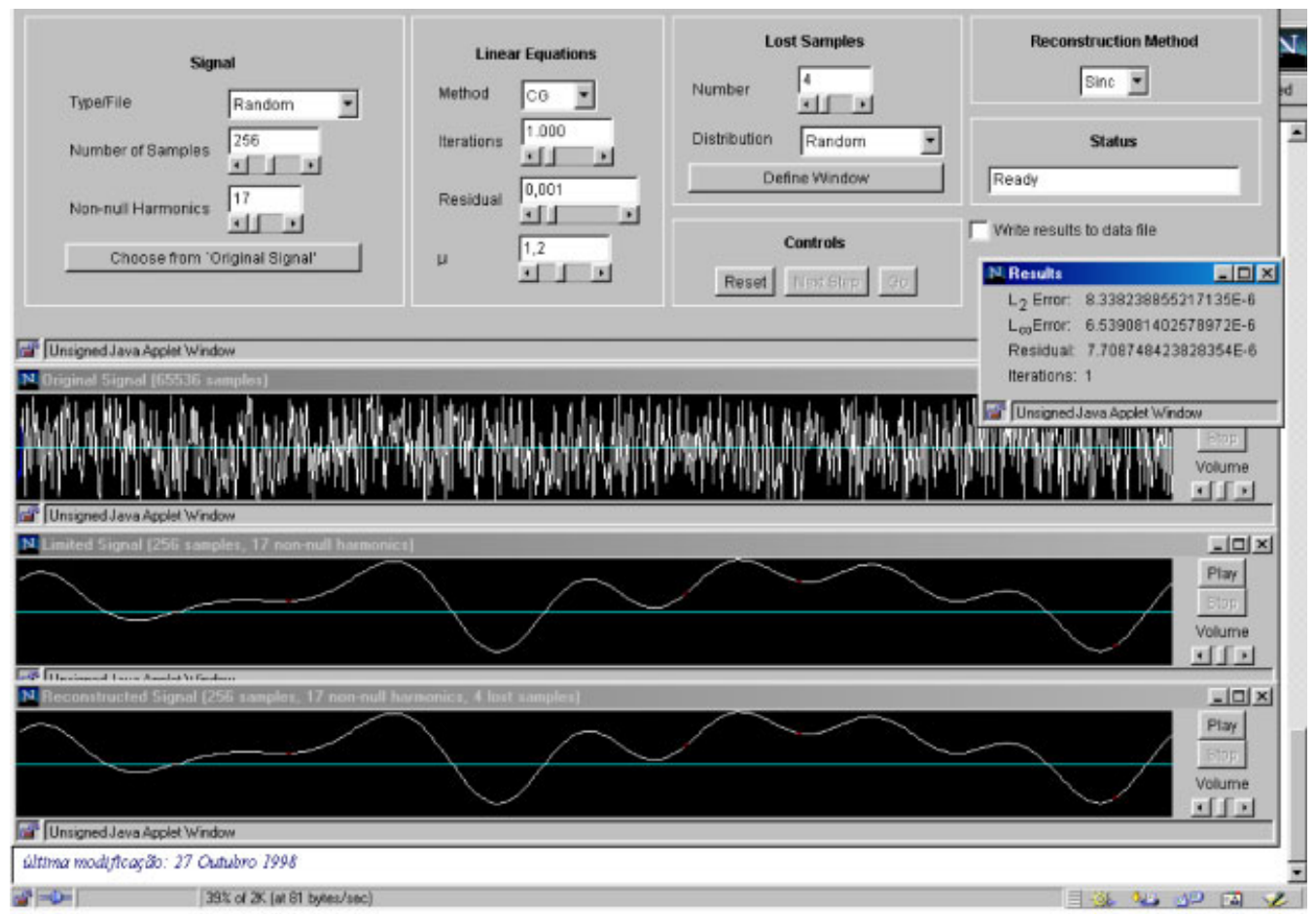

Figure 1 Signal applet general aspect. [Color figure can be viewed in the online issue, which is available at www.interscience.wiley.com.] 


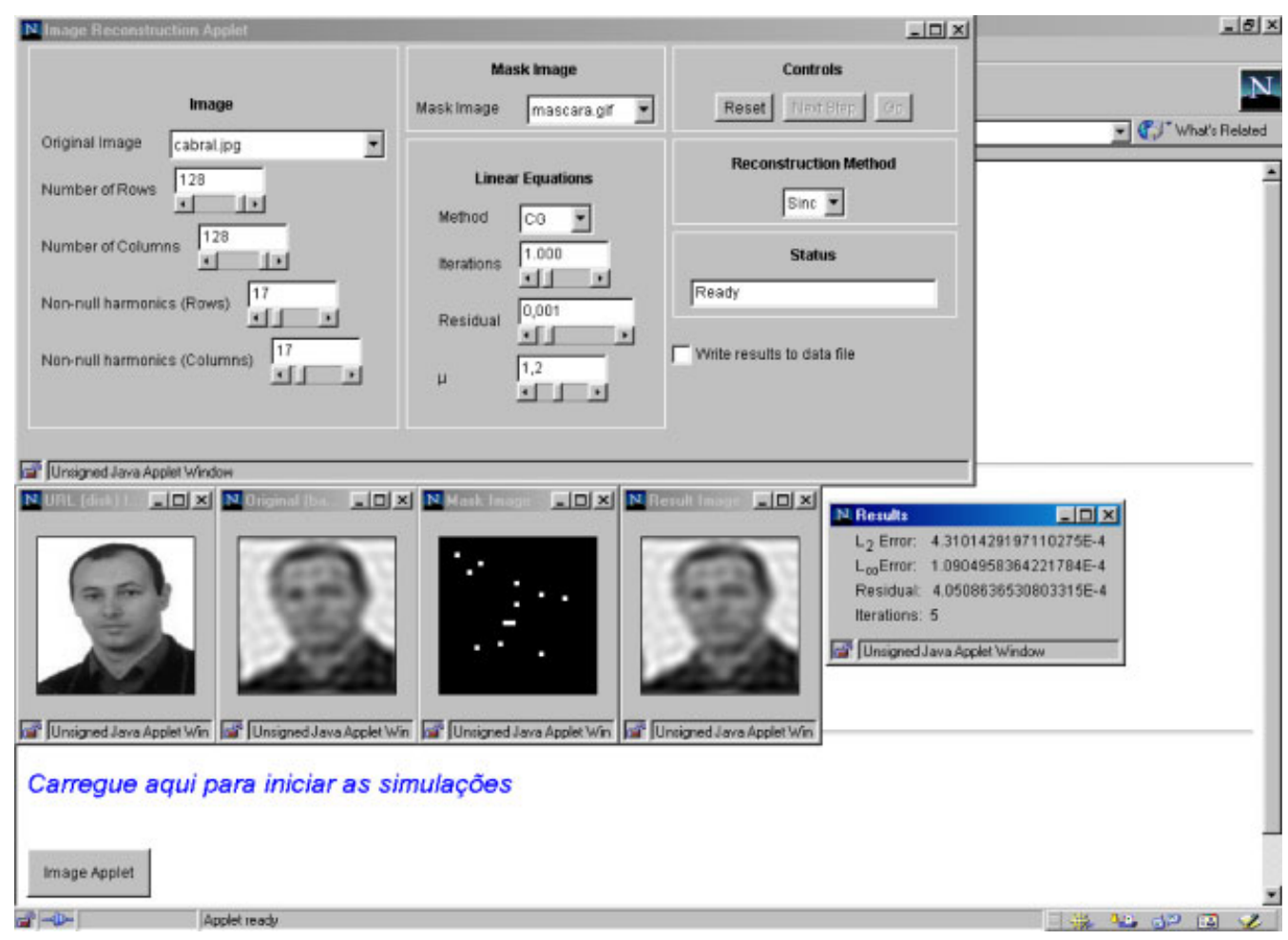

Figure 2 Image applet general aspect. [Color figure can be viewed in the online issue, which is available at www.interscience.wiley.com.]

The student may also control the number of lost samples and its distribution, which has a crucial impact on the numerical stability of the problem.

The final result may be attained step-by-step or in a single step. The student may evaluate the reconstruction result in two ways: by visualizing the intermediate (or final) results on the screen, or by listening to it on a loudspeaker (this interpretation may be very valuable when recovering music records). The results can be saved in an ASCII file that may be imported from: http://www.utad.pt/ mcabral/reconstrucao1d/ data/ for post-processing.

A full description of the file format and a complete user manual are also available. Figure 1 gives a general view of the applet.

\section{Image Reconstruction Applet}

This applet was developed along the same ideas and roughly possesses the same characteristics. The student can use its own images for test, by filling in an appropriate form. The image size (the number of rows and columns) and the two oversampling parameters (by rows and by columns) may also be controlled. The images can be in any format supported by the Java version.
The number of lost pixels and their locations are controlled by mask images. These images can be submitted in the same way as the original ones, that is, by filling in a form. In these images, the pixels given non-null values correspond to the unknown pixels in the original image.

Just as in the one-dimensional case, it is possible to select the method for solving the linear equations and the set of associated options. The final result can be reached step-by-step or in a single step, and the user may also choose to save the reconstruction quality results. Figure 2 shows a general aspect of the applet.

\section{CONCLUSIONS}

Signal and image reconstruction tasks are among the problems that an electrical engineer most often faces. An undergraduate course in the subject not only provides the student with relevant know-how concerning the problems, but may also be of considerable value in understanding how a background in Fourier and numerical analysis can be applied in the field, to concrete restoration problems.

In this context, we felt a need for platform independent tools that could be easily used inside or outside the classroom, and at the same time were easy 
to update and maintain. The applets that we have described allow the student to try several algorithms on their own data (signals or images), and then compare the results in an easy-to-use environment that is similar to a laboratory, from any computer system, and using their favorite browser.

The applets can be used by the students in their daily work, inside or outside the classroom, and have proved to be valuable tools for teaching signal and image reconstruction and understanding and comparing the very rich variety of associated computational methods.

\section{REFERENCES}

[1] J. J. Benedetto and P. J. S. G. Ferreira, Eds., Modern sampling theory: Mathematics and applications, Boston, Birkhäuser, 2001.

[2] P. L. Butzer, W. Engels, S. Ries, and R. L. Stens, The Shannon sampling series and the reconstruction of signals in terms of linear, quadratic, and cubic splines, SIAM J Appl Math 46 (1986), 299-323.

[3] P. J. S. G. Ferreira, Incomplete sampling series and the recovery of missing samples from oversampled bandlimited signals, IEEE Trans Signal Process 40 (1992), 225-227.

[4] P. J. S. G. Ferreira, Interpolation and the discrete Papoulis-Gerchberg algorithm, IEEE Trans Signal Process 42 (1994), 2596-2606.

[5] P. J. S. G. Ferreira, Noniterative and faster iterative methods for interpolation and extrapolation, IEEE Trans Signal Process 42 (1994), 3278-3282.

[6] P. J. S. G. Ferreira, The stability of a procedure for the recovery of lost samples in band-limited signals, Signal Process 40 (1994), 195-205.

[7] P. J. S. G. Ferreira, The duality of two recent image interpolation methods. In: Proceedings of the Third IEEE International Conference on Image Processing, ICIP-96, Vol. III, Lausanne, Switzerland, September 1996, pp 735-738.

[8] P. J. S. G. Ferreira, New algorithms for band-limited interpolation and extrapolation: A synthetic view. In:
Signal Processing $\mathrm{X}$-Theories and Applications, Proceedings of EUSIPCO-2000, X European Signal Processing Conference, Vol. IV, Tampere, Finland, September 2000, pp 2021-2024.

[9] P. J. S. G. Ferreira, Iterative and noniterative recovery of missing samples for 1-D band-limited signals. In: F. A. Marvasti, Ed., Sampling theory and practice, Boston, Plenum, 2001, pp 235-282.

[10] P. J. S. G. Ferreira and A. J. Pinho, Errorless restoration algorithms for band-limited images. In: Proceedings of the First IEEE International Conference on Image Processing, ICIP-94, Vol. III, Austin, TX, November 1994, pp 157-161.

[11] R. W. Gerchberg, Super-resolution through error energy reduction, Opt Acta 21 (1974), 709-720.

[12] K. Gröchenig, A discrete theory of irregular sampling, Linear Algebra Appl 193 (1993), 129-150,

[13] M. C. Jones, The discrete Gerchberg algorithm, IEEE Trans Acoust Speech Signal Process ASSP-34 (1986), 624-626.

[14] R. J. Marks II, Introduction to Shannon sampling and interpolation theory, New York, Springer-Verlag, 1991.

[15] A. Papoulis, A new algorithm in spectral analysis and band-limited extrapolation, IEEE Trans Circuit Syst CAS-22 (1975), 735-742.

[16] M. J. C. S. Reis and P. J. S. G. Ferreira, Minimum dimension image interpolation: Analytical results. In: NORSIG-96, 1996 IEEE Nordic Signal Processing Symposium, Espoo, Finland, September 1996, pp 87-90.

[17] M. J. C. S. Reis, P. J. S. G. Ferreira, and S. F. S. P. Soares, Linear combinations of b-splines as generating functions for signal approximation (in press).

[18] M. J. C. S. Reis and P. J. S. G. Ferreira, Reconstrução de sinal e imagem: Conjunto de programas para teste de diferentes algoritmos, Res Electrón Telecomun 3 (2003), 813-817.

[19] T. Strohmer, Efficient methods for digital signal and image reconstruction from nonuniform samples, $\mathrm{PhD}$ thesis, Institut für Mathematik der Universität Wien, November 1993.

[20] D. C. Youla, Generalized images restoration by the method of alternating orthogonal projections, IEEE Trans Circuits Syst CAS-25 (1978), 694-702.

\section{BIOGRAPHIES}

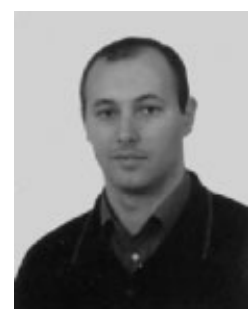

Manuel J. Cabral S. Reis received the $\mathrm{PhD}$ degree in electrical engineering and the MSc degree in electronics and telecommunications from the University of Aveiro, Portugal. Currently, he is a professor auxiliar in the Department of Engineering, University of Trás-os-Montes e Alto Douro, where he is the director of the MSc course in informatics. His research interests are in the area of signal processing, and include modeling and approximation, and problems such as sampling, interpolation, and signal reconstruction.

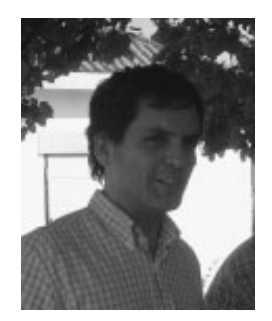

Paulo J. S. G. Ferreira received the $\mathrm{PhD}$ degree in electrical engineering from the University of Aveiro, Portugal, where he is currently a professor catedrático in the Department of Electronics and Telecommunications. He was an associate editor of the journal IEEE Transactions on Signal Processing and is on the editorial board of Sampling Theory in Signal and Image Processing and Journal of Applied Functional Analysis. He coedited, with John J. Benedetto, the book Modern Sampling Theory: Mathematics and Applications. His research interests are in the area of linear and nonlinear signal processing. 